\title{
ANÁLISE ESTRUTURAL E USO MÚLTIPLO DE ESPÉCIES ARBÓREAS EM FLORESTAS MANEJADAS NO MÉDIO VALE DO RIO CURUÁ-UNA, PARÁ
}

\author{
Diego dos Santos Vieira ${ }^{1 *}$, João Ricardo Vasconcellos Gama², Márcio Leles Romarco de Oliveira ${ }^{3}$, \\ Renato Bezerra da Silva Ribeiro ${ }^{2}$ Gerais, Brasil - diegovieir4@gmail.com
${ }^{2}$ Universidade Federal do Oeste do Pará, Instituto de Biodiversidade e Florestas, Santarém, Para, Brasil - jrvgama@gmail.com, forest_engineer@gmail.com
${ }^{3}$ Universidade Federal dos Vales do Jequitinhonha e Mucuri, Departamento de Engenharia Florestal, Diamantina, Minas Gerais, Brasil - marcioromarco@gmail.com \\ ${ }^{1 *}$ Universidade Federal dos Vales do Jequitinhonha e Mucuri, Programa de Pós-Graduação em Ciência Florestal, Diamantina, Minas
}

Recebido para publicação: 05/03/2014 - Aceito para publicação: 27/03/2015

\begin{abstract}
Resumo
O objetivo deste estudo foi avaliar alterações florísticas e estruturais ocorridas em áreas de Floresta Ombrófila Densa no Pará, em decorrência das atividades de exploração de impacto reduzido. Para isso, foram utilizadas duas áreas de floresta não manejada $\left(\mathrm{FNM}_{1}\right.$ e $\left.\mathrm{FNM}_{2}\right)$ e duas de floresta manejada $\left(\mathrm{FM}_{1}\right.$ e $\mathrm{FM}_{2}$ ), explorada há quatro anos, com intensidade de corte de $30 \mathrm{~m}^{3}$.ha ${ }^{-1}$. Utilizou-se amostragem estratificada, com alocação de 24 parcelas de 28 x 350 m, sendo 12 na $F_{1}$ e 12 na $F_{N M}$, e 20 parcelas de $50 \times 200 \mathrm{~m}$, sendo nove na $\mathrm{FM}_{2}$ e 11 na $\mathrm{FNM}_{2}$, mensurando-se todos os indivíduos com DAP $\geq 10 \mathrm{~cm}$. O delineamento foi inteiramente casualizado, com número diferente de repetições. No período de quatro anos após a exploração, ocorreram alterações não significativas na composição florística e abundância de indivíduos, no entanto os índices de Shannon calculados para as áreas diferiram significativamente. A espécie de maior valor de importância na $\mathrm{FM}_{1}$ e $\mathrm{FNM}_{1}$ foi Rinorea guianensis Aubl. e na $\mathrm{FM}_{2}$ e $\mathrm{FNM}_{2}$ foram Licania kunthiana Hook.f. e Richardella macrophylla (Lam.) Aubrév., respectivamente. Após quatro anos da colheita, foram identificadas diferenças significativas apenas no volume total e na dominância absoluta entre $\mathrm{FM}_{2}$ e $\mathrm{FNM}_{2}$.

Palavras-chave: Fitossociologia; Amazônia; manejo florestal.
\end{abstract}

\begin{abstract}
Structural analysis and multiple use of tree species in managed forest, Middle River Valley of Curuá-una, Pará. This research aimed to evaluate the floristic and structural changes in areas of dense rain forest in Pará, due to the reduced impact logging activities. Inorder to that, two areas of unmanaged forest were used $\left(\mathrm{FNM}_{1}\right.$ and $\left.\mathrm{FNM}_{2}\right)$, and two of managed forest $\left(\mathrm{FM}_{1}\right.$ and $\left.\mathrm{FM}_{2}\right)$, explored for four years with intensity of cut $30 \mathrm{~m}^{3} \cdot \mathrm{ha}^{-1}$. A stratified sampling was used with allocation of 24 plots of $28 \mathrm{~m} \mathrm{x} 350 \mathrm{~m}, 12$ in the $\mathrm{FM}_{1}$ and 12 in the $\mathrm{FNM}_{1}$, and 20 plots of $50 \mathrm{~m}$ x $200 \mathrm{~m}, 9$ in the $\mathrm{FM}_{2}$ and 11 in the $\mathrm{FNM}_{2}$, we measured all individuals with $\mathrm{DBH} \geq 10 \mathrm{~cm}$. The lineation was completely randomized with different number of repetitions. In the period of four years after forest harvest, non-significant changes occurred in the floristic composition and abundance of individuals, however, the Shannon-Weaver indices calculated for the areas differed significantly. The species with the highest importance value in $\mathrm{FM}_{1}$ and $\mathrm{FNM}_{1}$ was Rinorea guianensis Aubl. and in $\mathrm{FM}_{2}$ and $\mathrm{FNM}_{2}$ were Licania kunthiana Hook.f. and Richardella macrophylla (Lam.) Aubrév., respectively. After four years of harvests, significant differences were found only in total volume and absolute dominance between $\mathrm{FM}_{2}$ and $\mathrm{FNM}_{2}$.

Keywords: Phytosociology; Amazon; Forest management.
\end{abstract}

\section{INTRODUÇÃO}

A Amazônia é formada por um conjunto de ecossistemas que suportam diferentes sistemas de produção agrícola, pecuário e madeireiro. Esse panorama tem chamado a atenção e despertado o interesse da sociedade científica para o desenvolvimento de pesquisas que possam minimizar o efeito da ação

FLORESTA, Curitiba, PR, v. 45, n. 3, p. 465 - 476, jul. / set. 2015.

Vieira, D. dos S. et al.

ISSN eletrônico 1982-4688 / ISSN impresso 0015-3826

DOI: $10.5380 /$ rf.v45i3.35584 
antrópica, bem como criar estratégias para uso e manejo dos recursos naturais de forma sustentável, já que eles vêm sendo explorados sem adoção de critérios mínimos de sustentabilidade (PINTO et al., 2002).

Uma das estratégias criadas pelo Governo Federal foi a exigência do uso de Planos de Manejo Florestal Sustentável (PMFS), com intensidade de corte de $30 \mathrm{~m}^{3} \cdot \mathrm{ha}^{-1}$ em ciclos de 25 a 35 anos, para áreas que preveem o emprego de máquinas para o arraste de toras, e de $10 \mathrm{~m}^{3} \cdot \mathrm{ha}^{-1}$ com ciclos de corte inicial de10 anos para aquelas que não as utilizam. Desse modo, a adoção desses planos faria com que houvesse uma produção contínua de produtos e serviços florestais sem a indevida redução de seus valores intrínsecos e produtivos. No entanto, no estado do Pará, assim como no restante da Amazônia, as experiências de manejo florestal têm mostrado que muitos dos PMFS atualmente em andamento apresentam sérios problemas técnicos e de execução, ou seja, são desenvolvidos sem o emprego de técnicas de Exploração de Impacto Reduzido (EIR) e posterior aplicação de tratamentos silviculturais, resultando em impactos severos ao ecossistema florestal e, consequentemente, prejuízos às produções futuras (INSTITUTO BRASILEIRO DO MEIO AMBIENTE E DOS RECURSOS NATURAIS RENOVÁVEIS (IBAMA), 2007).

Na região do médio vale do rio Curuá-Una, diversas empresas madeireiras atuavam de maneira irregular, reduzindo a cobertura e a diversidade florestal antes mesmo que se tivesse o conhecimento da riqueza natural existente. No final da década de 1990, com a abertura de vias de acesso nessa região, o Instituto de Colonização e Reforma Agrária (INCRA), prefeituras e empresas madeireiras criaram projetos de assentamentos (PA's), com o objetivo de enfatizar o manejo florestal múltiplo e sistemas agroflorestais (GAMA et al., 2011). Assim, os lotes individuais desses assentamentos rurais podem ser explorados e oferecer produtos florestais madeireiros, não madeireiros e serviços ambientais, além de contribuir para mudanças no pensamento de aproveitamento unilateral da floresta, considerando que 50\% de uma propriedade rural constituam reserva legal, as quais podem ser manejadas para o aproveitamento comercial dos recursos florestais (ALMEIDA et al., 2012). No ano de 2006, por meio de contrato empresarial, a empresa de Manejo Florestal e Prestação de Serviço Ltda. (MAFLOPS) realizou o manejo florestal nesses lotes através do uso técnicas de EIR, colhendo em média $17,0 \mathrm{~m}^{3} \cdot \mathrm{ha}^{-1}$.

Apesar disso, não se tem conhecimento dos danos causados à composição florística, diversidade e estrutura de áreas submetidas à exploração florestal nesses PA's. Sendo assim, as atividades de EIR baseadas no uso de planos de manejo, por si só, não garantem a produção contínua de produtos florestais, sendo necessário conhecer e quantificar os impactos dessas atividades sobre a vegetação remanescente, na tentativa de eliminá-los ou reduzi-los ao mínimo, e assim garantir a conservação dos recursos florísticos e hídricos (MARTINS et al., 2003). Avaliações dessa natureza permitem a prescrição de medidas mitigadoras e ações que irão atuar viabilizando a execução de um plano de manejo com um mínimo impacto à comunidade vegetal.

Nesse contexto, o objetivo deste estudo foi analisar a fitossociologia e descrever o uso das principais espécies arbóreas no médio vale do rio Curuá-Una. Para tanto, foram discutidas as mudanças ocorridas na composição florística, diversidade e estrutura de áreas submetidas à exploração florestal, em comparação com áreas de floresta não manejada, de maneira a comprovar se o manejo florestal aplicado foi de baixo impacto, em relação às variáveis florísticas e estruturais da floresta não manejada.

\section{MATERIAL E MÉTODOS}

\section{Área de estudo}

O estudo foi realizado na região oeste do Pará, entre os municípios de Placas e Santarém, onde foram inventariadas quatro áreas de florestas, duas áreas com Manejo Florestal (FM) e duas sem manejo

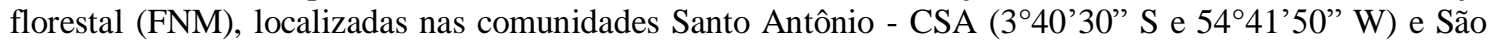

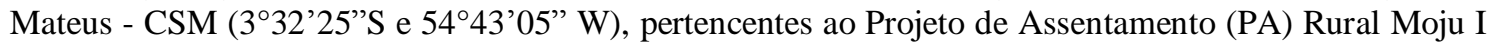
e II. Considerou-se como floresta manejada a área na qual houve interferência antrópica através do manejo de impacto reduzido, realizado em 2006, por meio de contrato empresarial, e floresta não manejada como área sem qualquer intervenção.

O clima da região, conforme Köppen, é classificado como Ami, ou seja, tropical úmido com variação térmica anual inferior a $5{ }^{\circ} \mathrm{C}$ e temperatura média anual de $25,5^{\circ} \mathrm{C}$, umidade relativa média do ar de $88 \%$ e precipitação pluviométrica anual de $1.820 \mathrm{~mm}$ (FUNDO DE DESENVOLVIMENTO E AÇÃO 
COMUNITÁRIA (FUNDAC), 2005). As áreas possuem a mesma tipologia, Floresta Ombrófila Densa de Terra Firme. O dossel é denso, fechado e compacto, situado entre 25 e $30 \mathrm{~m}$ de altura, sendo comuns indivíduos de Bertholletia excelsa Bonpl. (castanheira-do-brasil), Dinizia excelsa Ducke (angelimvermelho), Hymenaea courbaril L. (jatobá), Manilkara huberi (Ducke) A. Chev (maçaranduba) e Handroanthus serratifolius (Vahl) S. Grose (ipê-amarelo) nos estratos emergentes.

O manejo, pela extração seletiva de madeira, foi realizado pela empresa de Manejo Florestal e Prestação de Serviço Ltda. no ano de 2006, que colheu em média $17,0 \mathrm{~m}^{3} \cdot \mathrm{ha}^{-1}$ de madeira nas florestas manejadas, considerando-se o diâmetro a $1,30 \mathrm{~m}$ (DAP) maior ou igual a $50 \mathrm{~cm}$. O ciclo de corte instituído na área foi de 25 anos. Os dados de campo para este estudo foram coletados antes (2005) e após (2010) a realização das atividades florestais.

\section{Coleta de dados}

Para realização do inventário florestal, empregou-se amostragem estratificada com alocação de 42 unidades amostrais de forma sistemática, com múltiplos inícios aleatórios. O número de parcelas foi planejado para atender um erro de amostragem de até $10 \%$ para o volume de fuste, com $95 \%$ de probabilidade (IBAMA, 2007). Dessa forma, foram instaladas, em delineamento inteiramente casualizado, com número diferente de repetições, no sentido leste-oeste, 24 parcelas de $28 \times 350 \mathrm{~m}$ na CSA, sendo 12 na $F_{1}$ e 12 na $F_{N M}$, e 20 parcelas de 50 × 200 m na CSM, sendo 9 na $F_{2}$ e 11 na $\mathrm{FNM}_{2}$. A distância entre as áreas nas quais foram instaladas as unidades amostrais foi de aproximadamente $6 \mathrm{~km}$. O limite de inclusão das árvores na amostragem foi de diâmetro a 1,30 m (DAP) maior ou igual a $10 \mathrm{~cm}$, considerando as seguintes classes de tamanho (CT) e tamanhos de parcelas, a saber: $C T$ 1: $10 \mathrm{~cm} \leq$ DAP < $30 \mathrm{~cm}$ em subparcelas de $28 \times 100 \mathrm{~m}$ e $50 \times 20 \mathrm{~m} ; C T 2: 30 \mathrm{~cm} \leq$ DAP $<50 \mathrm{~cm}$ em subparcelas de $28 \times 250 \mathrm{~m}$ e $50 \times 40 \mathrm{~m}$; e $C T 3:$ DAP $\geq 50 \mathrm{~cm}$ nas parcelas de $28 \times 350 \mathrm{~m}$ e 50 x 200 m. Nos três níveis de inclusão, foram registrados os nomes locais das espécies, DAP e altura total de cada árvore.

As espécies foram identificadas em campo pelo nome regional, e aquelas que suscitaram dúvidas tiveram sua determinação taxonômica feita por especialistas, por comparações no herbário da Universidade Federal do Oeste do Pará. O sistema de classificação taxonômica adotado foi APG III (ANGIOSPERM PHYLOGENY GROUP (APG), 2009). Os autores das espécies foram confirmados no banco de dados do Missouri Botanical Garden (MISSOURI BOTANICAL GARDEN, 2014). O uso múltiplo das espécies foi verificado por meio de entrevistas com comunitários e classificado nos seguintes grupos: medicinal, madeireiro, alimentação humana, alimentação animal e produção de carvão.

\section{Análise de dados}

Os índices de similaridade de Sorensen (SO), diversidade de Shannon (H'), equabilidade de Pielou (J) e Quociente de Mistura de Jentsch (QM) foram estimados segundo Brower e Zar (1984). O padrão de distribuição espacial foi analisado pela relação entre a variância $\left(\mathrm{S}^{2}\right)$ e a média $(\mathrm{M})$ do número de árvores por unidade amostral. Os parâmetros fitossociológicos da estrutura horizontal e vertical foram estimados de acordo com Souza e Soares (2013). O volume de fuste comercial com casca de árvores em pé da $i$-ésima espécie $\left(V c_{i}\right)$ foi estimado utilizando-se o fator de forma igual a 0,7 .

Para estimar a posição sociológica absoluta das espécies na floresta, foram utilizados três estratos, conforme o critério recomendado por Souza e Soares (2013).

- Estrato Inferior $\left(\mathrm{E}_{1}\right)$ : Árvore com $H t<(\bar{H}-1 s)$;

- Estrato Médio $\left(\mathrm{E}_{2}\right)$ : Árvore com $(\bar{H}-1 s) \leq H t<(\bar{H}+1 s)$;

- Estrato Superior $\left(\mathrm{E}_{3}\right)$ : Árvore com $H t \geq(\bar{H}+1 s)$.

em que: $\bar{H}=$ média das alturas totais $(\mathrm{Ht})$ dos indivíduos amostrados; s = desvio padrão das alturas totais (Ht) dos indivíduos amostrados.

As variáveis florísticas e estruturais foram comparadas por meio dos quatro tratamentos. As variáveis densidade, dominância e volume foram analisadas por meio de análise de variância (ANOVA), sendo aplicado o teste Scott-Knott com 95\% de probabilidade para comparar as médias dos tratamentos. A 
comparação entre distribuições diamétricas para FM e FNM nas comunidades CSA e CSM foi realizada por meio do teste Kolmogorov-Smirnov (KS). Para efeito de comparação da diversidade entre os sítios, utilizouse $o$ teste $t$ de Hutchinson (2), a 95\% de probabilidade (BROWER; ZAR, 1984), por meio da expressão:

$$
t=\frac{H_{1}+H_{2}}{\sqrt{\widehat{\sigma}_{H_{1}}^{2}+\widehat{\sigma}_{H_{2}}^{2}}}
$$

O estimador para a variância (3) e o grau de liberdade (4) do teste $t$ de Hutchinson foi definido por:

$$
\hat{\sigma}_{H}^{2}=\frac{\sum p_{i} \cdot\left[n\left(p_{i}\right)\right]^{2}-\frac{\sum p_{i} \cdot \operatorname{Ln}\left(p_{i}\right)}{N}}{N^{2}}
$$

$$
g l=\frac{\left(\widehat{\sigma}_{H_{1}}^{2}+\widehat{\sigma}_{H_{2}}^{2}\right)^{2}}{\frac{\left(\widehat{\sigma}_{H_{1}}^{2}\right)^{2}}{N_{1}}+\frac{\left(\widehat{\sigma}_{H_{2}}^{2}\right)^{2}}{N_{2}}}
$$

em que: $\mathrm{N}_{1}$ e $\mathrm{N}_{2}$ são números de indivíduos amostrados nas comunidades.

Os dados passaram pelo teste de normalidade de Shapiro-Wilk e de homogeneidade de variância; quando necessário, aplicou-se transformação logarítmica e quártica. As variáveis que não atingiram normalidade foram analisadas por meio da estatística não paramétrica pelo Teste de Kruskal-Wallis a $95 \%$ de probabilidade. A tabulação e o processamento de dados foram realizados com uso do software Biostat 5.0.

\section{RESULTADOS E DISCUSSÃO}

\section{Composição florística}

Para as quatro florestas amostradas, foram encontrados 4.262 indivíduos com DAP $\geq 10 \mathrm{~cm}$, distribuídos em 252 espécies e 44 famílias. Foram identificadas 173, 183, 134 e 146 espécies, respectivamente, nos tratamentos $\mathrm{FM}_{1}, \mathrm{FNM}_{1}, \mathrm{FM}_{2}$ e $\mathrm{FNM}_{2}$ (Tabela 1). Na Floresta Nacional do Tapajós - FNT (PA), avaliando indivíduos com DAP $\geq 10 \mathrm{~cm}$, Espírito-Santo et al. (2005) registraram 180 espécies em floresta primária, considerando uma amostra de 35 parcelas de 0,25 ha distribuídas em diferentes locais da FNT.

Dentre as famílias que apresentaram maior número de espécies, destacam-se as seguintes: Fabaceae (61), Sapotaceae (18), Moraceae (13), Lecythidaceae (13) e Apocynaceae (10). Gonçalves e Santos (2008), Almeida et al. (2012) e Ribeiro et al. (2013), em áreas de floresta manejada e não manejada nos municípios de Santarém e Placas (PA), respectivamente, considerando DAP $\geq 10 \mathrm{~cm}$, também atribuíram a Fabaceae o maior número de espécies, seguida por Sapotaceae e Lecythidaceae. Verificou-se ainda que, das 252 espécies, $81(32,14 \%)$ apresentaram maior plasticidade em distribuição, atingindo $100 \%$ de ocorrência entre as áreas. Por outro lado, 27\% das espécies foram exclusivas de, pelo menos, uma área, podendo ser considerada exclusiva de seu local de ocorrência.

Tabela 1. Índices de diversidade florística e estrutural da floresta, considerando indivíduos com DAP $\geq$ $10 \mathrm{~cm}$, em área de Floresta Ombrófila Densa no Médio Vale do Rio Curuá-Una, Pará, Brasil.

\begin{tabular}{|c|c|c|c|c|}
\hline Parâmetros & FM $_{1}$ & FNM $_{1}$ & $\mathbf{F M}_{2}$ & FNM $_{2}$ \\
\hline Número de famílias & 39 & 41 & 35 & 35 \\
\hline Volume $\left(\mathrm{m}^{3} \cdot \mathrm{ha}^{-1}\right)$ & 266,8 & 318,5 & 324,1 & 389,7 \\
\hline Riqueza de espécies (S) & 173 & 183 & 134 & 146 \\
\hline Densidade absoluta $\left(\mathrm{n}^{\circ}\right.$ ind. $\left.\mathrm{ha}^{-1}\right)$ & 254,8 & 298,7 & 472,56 & 508,0 \\
\hline Área basal $\left(\mathrm{m}^{2} \cdot \mathrm{ha}^{-1}\right)$ & 18,5 & 23,1 & 26,26 & 28,89 \\
\hline Diversidade máxima $\left(\mathrm{H}_{\text {máx }}\right)$ & 5,15 & 5,21 & 4,90 & 4,98 \\
\hline Índice de Shannon (H') & 3,89 & 4,52 & 2,93 & 4,19 \\
\hline Equabilidade de Pielou (J) & 0,86 & 0,86 & 0,84 & 0,86 \\
\hline Coeficiente de Mistura de Jentsch (QM) & $1: 7$ & $1: 8$ & $1: 5$ & $1: 6$ \\
\hline
\end{tabular}

Table 1. Floristic diversity and forest structure index, for individuals with $\mathrm{DBH} \geq 10 \mathrm{~cm}$, in an area of dense rain forest in the Middle River Valley of Curuá-Una, Pará, Brazil.

$\mathrm{FM}_{1}$ : floresta manejada e $\mathrm{FNM}_{1}$ : floresta não manejada na comunidade Santo Antônio; $\mathrm{FM}_{2}$ e FNM $\mathrm{FNa}_{2}$ comunidade São Mateus. 
O índice de diversidade de Shannon variou de 4,52 a 2,93, sendo semelhante ao índice calculado por Alves e Miranda (2008), Almeida et al. (2012) e Ribeiro et al. (2013) em áreas de floresta de terra firme manejadas e não manejadas em Santarém, Almeirim e Placas, oeste do Pará, demonstrando que as áreas em estudo, exceto $\mathrm{FM}_{2}$, possuem alta diversidade quando comparadas com outras florestas ombrófilas densas de terra firme no estado do Pará. Na CSA e CSM, observaram-se reduções na diversidade, contrastando a hipótese apresentada por Martins et al. (2003) de que haveria aumento de diversidade após a exploração florestal, ocasionada pela abertura de clareiras, no espaço de tempo decorrido entre a colheita florestal e a segunda amostragem.

$\mathrm{O}$ índice de equabilidade de Pielou (J) foi superior a 0,80, o que significa que não há a dominância de uma ou de um pequeno grupo de espécies nas comunidades, indicando alta heterogeneidade florística. O quociente de mistura (QM) variou de 1:6 a 1:8, sendo superior aos encontrados por Ximenes et al. (2011) em sítio manejado (1:4) e não manejado (1:5) na Floresta Nacional do Tapajós, em uma amostra de 3,7 ha (DAP $\geq 5 \mathrm{~cm}$ ), e inferior ao registrado por Francez et al. (2007) em floresta manejada no município de Paragominas, PA. Os resultados observados para $\mathrm{FM}_{1}$ e $\mathrm{FNM}_{1}$ aproximaram-se da afirmativa de Finol (1975) de que, em florestas naturais tropicais, o QM seria de, aproximadamente, nove indivíduos por espécie, indicando alta heterogeneidade.

$\mathrm{Na}$ análise do índice de Shannon, referente às quatro áreas, pode-se constatar que houve diferenças significativas, pelo teste $t$ de Hutchinson a $95 \%$ de probabilidade, entre e dentro de comunidades, concluindo-se que a diversidade nas áreas manejadas foi afetada pela colheita seletiva (Tabela 2).

Tabela 2. Médias florísticas e estruturais no Médio Vale do Rio Curuá-Una, Pará, Brasil.

Table 2. Floristic and structural averages in the Médio Vale do Rio Curuá-Una, Pará, Brazil.

\begin{tabular}{lcccccc}
\hline Tratamentos* & Fam. & S & H' & DA & G & $\mathbf{V}_{\mathbf{f}}$ \\
\hline $\mathrm{FM}_{1}$ & $39 \mathrm{a}$ & $173 \mathrm{a}$ & $3,89 \mathrm{a}$ & $254,8 \mathrm{a}$ & $18,5 \mathrm{a}$ & $266,7 \mathrm{a}$ \\
$\mathrm{FNM}_{1}$ & $41 \mathrm{a}$ & $183 \mathrm{a}$ & $4,52 \mathrm{~b}$ & $298,7 \mathrm{a}$ & $23,1 \mathrm{a}$ & $318,5 \mathrm{a}$ \\
$\mathrm{FM}_{2}$ & $35 \mathrm{~b}$ & $134 \mathrm{~b}$ & $2,93 \mathrm{c}$ & $472,5 \mathrm{~b}$ & $26,3 \mathrm{~b}$ & $324,1 \mathrm{a}$ \\
$\mathrm{FNM}_{2}$ & $35 \mathrm{~b}$ & $146 \mathrm{~b}$ & $4,19 \mathrm{~d}$ & $508,0 \mathrm{~b}$ & $28,8 \mathrm{c}$ & $389,8 \mathrm{~b}$ \\
\hline
\end{tabular}

Tratamentos com letras iguais na vertical não diferem estatisticamente entre si. $\mathrm{FM}_{1:}$ floresta manejada; $\mathrm{FNM}_{1}$ : floresta não manejada na comunidade Santo Antônio; $\mathrm{FM}_{2}$ e FNM 2 na comunidade São Mateus; Fam.: número de família; S: riqueza de espécies; $\mathrm{H}^{\prime}$ : índice de Shannon; DA: densidade em n.ha ${ }^{-1}$; $\mathrm{G}$ área basal $\mathrm{em} \mathrm{m}^{2}$.ha ${ }^{-1} ; \mathrm{V}_{\mathrm{f}}$ : volume de fuste $\mathrm{em}^{3}$.ha ${ }^{-1}$.

De maneira geral, as intervenções realizadas provocaram a diminuição no número de espécies e de famílias logo após a sua aplicação. Todavia, com o passar do tempo, é possível que a maior abertura no dossel tenha estimulado a entrada de novas espécies no povoamento, de tal forma que quatro anos depois da colheita florestal não foram constatadas diferenças significativas $(\mathrm{p}<0,05)$, pelo teste ScottKnott a 95\% de probabilidade, entre os tratamentos pertencentes à mesma comunidade, quanto aos valores de riqueza e número de famílias.

Quanto à distribuição espacial, observou-se que, na $\mathrm{FM}_{1}, 49$ espécies $(28,3 \%)$ ocorreram de forma aleatória na amostra, $32(18,5 \%)$ tenderam ao agrupamento e $46(26,6 \%)$ ocorreram de maneira agrupada. $\mathrm{Na} \mathrm{FNM}_{1}, 63$ espécies $(34,4 \%)$ apresentaram padrão agrupado, $46(25,1 \%)$ padrão aleatório e $29(15,8 \%)$ tenderam ao agrupamento. $\mathrm{Na} \mathrm{FM}_{2}$, predominaram dois tipos de padrões, aleatório e agrupado, com $35(26,1 \%)$ cada, e $15(11,2 \%)$ foram consideradas com tendência ao agrupamento. Na $\mathrm{FNM}_{2}, 47$ (32,2\%) prevaleceram com padrão de distribuição agrupado, 39 (26,7\%) de forma aleatória e $23(15,8 \%)$ foram consideradas com tendência ao agrupamento.

Através da análise espacial das espécies arbóreas estudadas, observou-se que o padrão de agregação das espécies foi afetado pela colheita florestal, mas algumas espécies com uso madeireiro no PA Moju I e II, como Aspidosperma desmanthum Benth. ex Mull. Arg., Iryanthera juruensis Warb., Jacaranda copaia (Aubl.) D. Don e Aniba megaphylla Mez, que apresentavam padrão agregado ou tendência à agregação $\left(P_{i} \geq 1,0\right)$ antes da exploração, passaram ao padrão aleatório $\left(P_{i}<1,0\right)$ após a colheita florestal. A mudança no padrão de distribuição espacial das espécies também foi observado por Martins et al. (2003) na colheita de florestas no Paraná (BR).

Nas áreas nas quais houve extração seletiva de madeira, $\mathrm{FM}_{1}$ e $\mathrm{FM}_{2}$, prevaleceu a distribuição espacial aleatória e agrupada, respectivamente, enquanto nas áreas nas quais não houve intervenção antrópica $\left(\mathrm{FNM}_{1}\right.$ e $\left.\mathrm{FNM}_{2}\right)$ predominou o padrão agrupado. De acordo com Matteucci e Colma (1982), o 
padrão de distribuição espacial agregado é o mais frequente em florestas tropicais, seguido do aleatório e do uniforme. Para espécies com padrão agregado, recomenda-se manter alguns indivíduos de tamanho comercial, para que continue a ocorrer a distribuição natural. Já para aquelas espécies com distribuição aleatória, devem-se manter indivíduos para o fornecimento de novos cortes e, principalmente, para garantir a perpetuidade da espécie na área.

Dentre as espécies que ocorreram de forma agrupada, destacam-se aquelas com potencial para uso medicinal: Carapa guianensis Aubl., Protium sp., Bertholletia excelsa Bonpl., Calophyllum brasiliense Cambess., Mouriria sagotiana Triana e Aniba canelilla (Kunt) Mez. Segundo Machado (2008), o manejo de produtos florestais não madeireiros é favorecido quando os indivíduos ocorrem de forma agrupada, devido à facilidade logística para coleta dos produtos não madeireiros oriundos dessas espécies.

As espécies com um único indivíduo na amostragem totalizaram 46, 45, 49 e 37, respectivamente, para $\mathrm{FM}_{1}, \mathrm{FNM}_{1}, \mathrm{FM}_{2}$ e $\mathrm{FNM}_{2}$. Essas espécies necessitam de maior amostragem na área e distribuição diferenciada de unidades amostrais, para uma melhor descrição do padrão de distribuição.

\section{Similaridade florística}

A análise do grau de semelhança florística entre os tratamentos, com base em todas as espécies, constatou que tratamentos pertencentes à mesma comunidade foram os mais similares ( $\mathrm{SO} \geq 66 \%$ ), com um nível de ligação em torno de $79 \%\left(\mathrm{FM}_{1}\right.$ e $\left.\mathrm{FNM}_{1}\right)$, seguidos por $\mathrm{FM}_{2}$ e $\mathrm{FNM}_{2}$, com nível de ligação de 78\% (Tabela 3). De acordo com Kent e Coker (1992), valores maiores ou iguais a 50\% indicam alta similaridade. Assim, segundo esse critério, a similaridade analisada entre as áreas pode ser considerada como alta.

Tabela 3. Similaridade florística de Sorensen entre os tratamentos, Médio Vale do Rio Curuá-Una, estado do Pará, Brasil.

Table 3. Sorensen floristic similarity between treatments, Médio Vale do Rio Curuá-Una, state of Pará, Brazil.

\begin{tabular}{lcccc}
\hline Tratamentos & $\mathbf{F M}_{\mathbf{1}}$ & $\mathbf{F N M}_{\mathbf{1}}$ & $\mathbf{F M}_{\mathbf{2}}$ & $\mathbf{F N M}_{\mathbf{2}}$ \\
\hline $\mathrm{FM}_{1}$ & - & - & - & - \\
$\mathrm{FNM}_{1}$ & 0,79 & - & - & - \\
$\mathrm{FM}_{2}$ & 0,67 & 0,66 & - & - \\
$\mathrm{FNM}_{2}$ & 0,69 & 0,66 & 0,78 & - \\
\hline $\mathrm{FM}_{1:}$ floresta manejada; & $\mathrm{FNM}_{1}$ : floresta não & manejada na comunidade Santo Antônio; & $\mathrm{FM}_{2}$ e $\mathrm{FNM}_{2}$ na \\
comunidade São Mateus.
\end{tabular}

A explicação para os altos valores de similaridade entre as áreas está relacionada à proximidade geográfica, que proporciona tipologia florestal e fatores edafoclimáticos mais homogêneos, tais como classe de solo, fertilidade do solo, altitude, precipitação e temperatura (GAMA et al., 2005).

\section{Distribuição diamétrica}

A distribuição do número de árvores por classe diamétrica, com amplitude de $10 \mathrm{~cm}$, seguiu o padrão característico das florestas inequiâneas, isto é, distribuição exponencial negativa, na forma de "J" invertido, que, segundo Vieira et al. (2014), é o comportamento típico de muitas florestas de terra firme da Amazônia Oriental (Figura 1).

Comparado as distribuições diamétricas para FM e FNM nas comunidades CSA e CSM, obtevese $\rho=0,699$ e $\rho=0,979$, respectivamente. Pelos resultados encontrados com o teste KolmogorovSmirnov, os valores das probabilidades ( $\rho$ ) calculadas foram superiores a 0,05 , verificando-se que não houve diferença significativa entre as distribuições diamétricas, o que permite concluir que a estrutura da floresta foi pouco alterada pelo manejo florestal.

\section{Análise estrutural}

A densidade de árvores estimada na $\mathrm{FM}_{1}$ foi de 254,8 ind.ha ${ }^{-1}$, com área basal de 18,5 m².ha $\mathrm{ha}^{-1} \mathrm{e}$ volume de fuste de $266,8 \mathrm{~m}^{3} \cdot \mathrm{ha}^{-1}$ (Tabela 1). Na FNM ${ }_{1}$, constataram-se 298,7 ind.ha ${ }^{-1}$, com área basal de $23,1 \mathrm{~m}^{2} \cdot \mathrm{ha}^{-1} \mathrm{e}$ volume de fuste de $318,5 \mathrm{~m}^{3} \cdot \mathrm{ha}^{-1}$ (Tabela 1). A altura dominante das árvores para $\mathrm{FM}_{1} \mathrm{e}$ $\mathrm{FNM}_{1}$ foi de $31,3 \mathrm{~m}$ e $29,9 \mathrm{~m}$, respectivamente. 

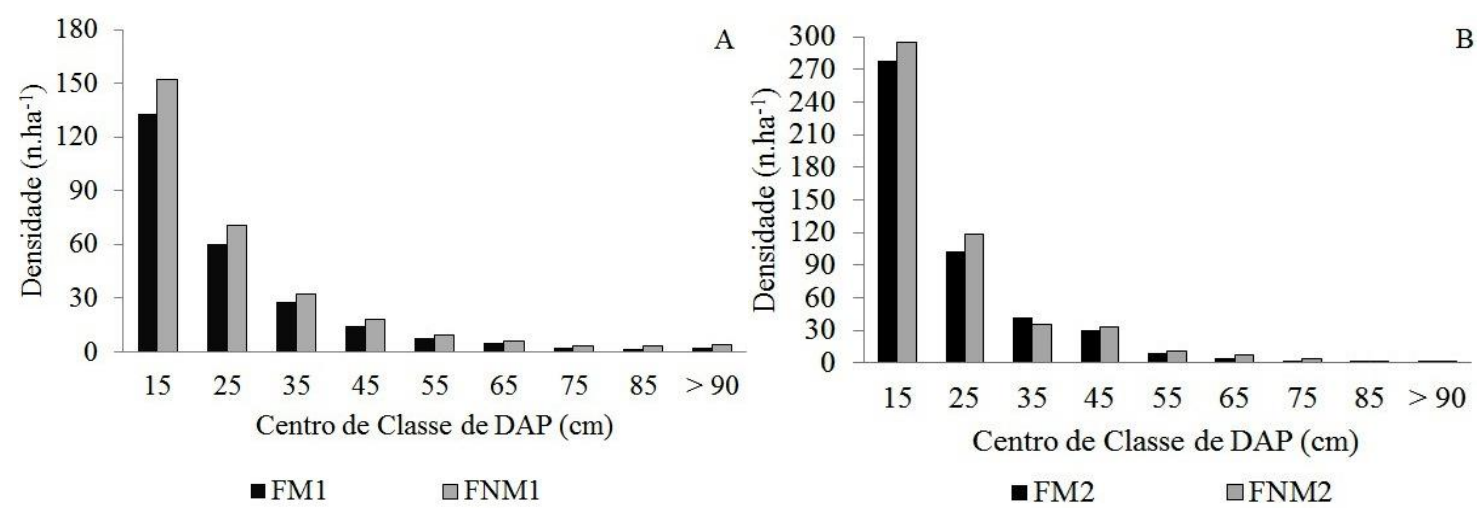

Figura 1. Distribuição diamétrica dos indivíduos de uma Floresta Ombrófila Densa nas Comunidades Santo Antônio [A] e São Matheus [B], Projeto de Assentamento Moju I e II, município de Santarém e Placas, estado do Pará.

Figure 1. Diametric distribution of individuals in a tropical rain forest in Santo Antônio Community [A] and São Matheus [B], Moju Settlement Project I and II, Municipalities of Santarém and Placas, State of Para.

Ao serem comparadas as condições de floresta, foram constatadas reduções de 14,7, 16,2 e 20\%, respectivamente, no número de indivíduos por hectare, volume de fuste e área basal, porém tais diferenças não foram significativas ( $\mathrm{p}>0,05)$, ficando, assim, evidente que o manejo florestal, ao diminuir a densidade absoluta, ativa e acelera os processos de dinâmica sucessional, retomando o crescimento, de forma que o estoque explorado ficou sendo reposto após a exploração, o que é uma característica importante para a escolha das espécies que podem ser manejadas.

Entre as 173 espécies identificadas na $\mathrm{FM}_{1}, 63$ apresentaram densidade absoluta (DA) igual ou superior a 1 ind.ha ${ }^{-1}$. As dez espécies mais abundantes (DA $\geq 5$ ) foram: Rinorea guianensis Aubl., Protium cf. heptaphyllum (Aubl) Marchand, Eschweilera coriacea (DC.) S.A.Mori, Richardella macrophylla (Lam.) Aubrév., Pouteria bilocularis (H. Winkler) Baehni, Sclerolobium sp., Sclerolobium paniculatum Vogel, Protium paniculatum var. riedelianum (Engl.) Daly, Inga sp., Nectandra sp. Juntas, representam 33,7\% da densidade absoluta total (Tabela 4). O padrão de distribuição espacial dessas espécies foi de tendência a agrupamento, exceto Sclerolobium paniculatum e Nectandra sp., com padrão aleatório.

Entre as 183 espécies identificadas na $\mathrm{FNM}_{1}, 75$ apresentaram DA $\geq 1$ ind.ha ${ }^{-1}$. As dez espécies mais abundantes (DA $\geq 6$ ) foram Rinorea guianensis Aubl., Eschweilera amazonica Knuth, Protium cf. heptaphyllum, Inga alba (Sw.) Willd., Pouteria guianensis Aubl., Sclerolobium sp., Franchetella anibaefolia (A.C. Smith.) Aubr., Endlicheria longicaudata (Ducke) Kosterm., Eschweilera coriacea (DC.) S.A.Mori e Protium paniculatum var. riedelianum (Engl.) Daly, que, juntas, contribuíram com $32,8 \%$ da densidade absoluta total. Essas espécies apresentaram-se distribuídas de forma agregada ou tendendo à agregação.

Para verificar a ocorrência das espécies nos diferentes estratos verticais da floresta, realizou-se análise da posição sociológica absoluta (PSA) e valor de importância ampliado (VIA). As 15 espécies mais importantes quanto a esses parâmetros, juntas, representaram 36,7 e $35,4 \%$ do VIA total da FM $_{1}$ e $\mathrm{FNM}_{1}$, respectivamente (Tabela 4). As árvores, nas quatro áreas estudadas, foram agrupadas em três estratos: estrato inferior (árvores que alcançavam altura menor que $11 \mathrm{~m}$ ), estrato médio (árvores com $11 \mathrm{~m} \leq \mathrm{H}_{\mathrm{t}}<23 \mathrm{~m}$ ) e estrato superior (árvores com $\mathrm{Ht}>23 \mathrm{~m}$ ).

As espécies que ocorreram em todos os estratos da floresta e obtiveram os maiores valores de PSA na $\mathrm{FM}_{1}$ foram: Protium cf. heptaphyllum, Richardella macrophylla, Manilkara huberi, Carapa guianensis, Nectandra sp., Protium paniculatum var.riedelianum, Pouteria caimito (Ruiz \& Pav.) Radlk. e Pouteria cladantha Sandwith. Espécies que estão presentes em todos os estratos asseguram sua representatividade na estrutura da comunidade vegetal, no entanto, as que não seguem essas normas podem não estar presentes na comunidade vegetal a longo prazo, por não se reproduzirem ou regenerarem no local, com exceção daquelas que são características dos estratos inferior e médio da floresta. 
Tabela 4. Estimativa dos parâmetros fitossociológicos das espécies com valor de importância ampliado (VIA) $\geq 1,5$ para os tratamentos $\mathrm{FM}_{1}$ e $\mathrm{FNM}_{1}$, município de Santarém, Pará.

Table 4. Estimation of phytosociological parameters of species with value larger importance (VIA) $\geq$ 1.5 for $\mathrm{FM}_{1}$ and $\mathrm{FNM}_{1}$ treatments, Municipality of Santarém, Pará.

\begin{tabular}{|c|c|c|c|c|c|c|c|c|}
\hline \multirow{2}{*}{ Espécies } & \multicolumn{2}{|c|}{$\mathbf{P}$} & \multicolumn{2}{|c|}{ FA $(\%)$} & \multicolumn{2}{|c|}{ DA $\left(\right.$ n.ha $\left.{ }^{-1}\right)$} & \multicolumn{2}{|c|}{ VIA (\%) } \\
\hline & $\mathbf{F M}_{1}$ & FNM $_{1}$ & $\mathbf{F M}_{1}$ & FNM $_{1}$ & $\mathbf{F M}_{1}$ & FNM $_{1}$ & $\mathbf{F M}_{1}$ & FNM $_{1}$ \\
\hline Rinorea guianensis Aubl. & AG & AG & 75,0 & 83,3 & 26,2 & 24,3 & 7,19 & 5,44 \\
\hline Protium cf. heptaphyllum (Aubl.) Marchand & AG & AG & 66,7 & 66,7 & 10,4 & 8,8 & 3,00 & 2,32 \\
\hline Eschweilera coriacea (DC.) S.A.Mori & AG & AG & 33,3 & 83,3 & 10,3 & 12,4 & 2,88 & 3,50 \\
\hline Pouteria bilocularis (H. Winkler) Baehni & TAG & AG & 100 & 91,7 & 6,0 & 4,3 & 2,76 & 1,72 \\
\hline Sclerolobium paniculatum Vogel & $\mathrm{AL}$ & $\mathrm{AL}$ & 100 & 91,7 & 5,3 & 4,8 & 2,49 & 1,72 \\
\hline Sclerolobium sp. & $A G$ & AG & 66,7 & 75,0 & 5,9 & 8,1 & 2,37 & 2,47 \\
\hline Richardella macrophylla (Lam.) Aubrév. & TAG & - & 83,3 & - & 6,6 & - & 2,07 & - \\
\hline Manilkara huberi (Ducke) Chev. & TAG & TAG & 91,7 & 100 & 3,5 & 6,0 & 1,87 & 3,08 \\
\hline Carapa guianensis Aubl. & AG & - & 66,7 & - & 4,6 & - & 1,84 & - \\
\hline Cassia scleroxylon Ducke & AG & - & 58,3 & - & 4,7 & - & 1,83 & - \\
\hline Nectandra sp. & $\mathrm{AL}$ & - & 91,7 & - & 5,0 & - & 1,80 & - \\
\hline Geissospermum sericeum Miers & TAG & - & 83,3 & - & 4,0 & - & 1,78 & - \\
\hline $\begin{array}{l}\text { Protium paniculatum var. riedelianum } \\
\text { (Engl.) Daly }\end{array}$ & AG & AG & 58,3 & 75,0 & 5,1 & 6,6 & 1,74 & 1,97 \\
\hline Pouteria caimito (Ruiz \& Pav.) Radlk. & AG & - & 66,7 & - & 3,9 & - & 1,53 & - \\
\hline Pouteria cladantha Sandwith & AG & - & 58,3 & - & 3,8 & - & 1,52 & - \\
\hline Lecythis jarana (Huber \& Ducke) A.C.Sm & - & TAG & - & 91,7 & - & 3,9 & - & 1,68 \\
\hline Eschweilera grandiflora (Aubl.) Sandwith & - & TAG & - & 83,3 & - & 4,5 & - & 1,75 \\
\hline Pouteria guianensis Aubl. & - & AG & - & 91,7 & - & 8,3 & - & 2,26 \\
\hline Franchetella anibaefolia (A.C. Smith.) Aubl. & - & AG & - & 66,7 & - & 7,8 & - & 2,28 \\
\hline Inga alba (Sw.) Willd. & - & AG & - & 58,3 & - & 8,6 & - & 2,09 \\
\hline Eschweilera amazonica Knuth & - & AG & - & 50,0 & - & 6,6 & - & 1,59 \\
\hline Endlicheria longicaudata (Ducke) Kosterm. & - & TAG & - & 75,0 & - & 6,6 & - & 1,58 \\
\hline Subtotal & - & - & 1.100 & 1.183 & 105,2 & 121,5 & 37 & 35 \\
\hline Total & - & - & 4.900 & 5.400 & 254,8 & 299 & 100 & 100 \\
\hline
\end{tabular}

$\mathrm{Na} \mathrm{FNM}_{1}$, Eschweilera coriacea, Pouteria bilocularis, Sclerolobium paniculatum, Sclerolobium sp., Richardella macrophylla, Manilkara huberi (Ducke) A. Chev., Carapa guianensis, Cassia scleroxylon Ducke, Nectandra sp., Geissospermum sericeum Miers, Pouteria cladantha, Lecythis jarana (Huber ex Ducke) A.C.Sm., Eschweilera grandiflora (Aubl.) Sandwith, Pouteria guianensis, Franchetella anibaefolia e Inga alba foram as espécies que ocorreram em todos os estratos da floresta e obtiveram os maiores valores de PSA e VIA.

Quanto à destinação das espécies na $\mathrm{FM}_{1}$, aquelas com os maiores valores de VIA (VIA $\geq 2,0 \%$ ) foram: Rinorea guianensis (espécie preferencial para produção de energia), Protium cf. heptaphyllum e Pouteria bilocularis (a madeira dessas espécies pode ser aproveitada em serraria e seus frutos consumidos pela fauna), Eschweilera coriacea (incluída na categoria de construções rústicas, por sua casca ser usada na fabricação de cordas); Sclerolobium paniculatum (utilizada em serraria e com potencial para recuperação de áreas degradadas), Sclerolobium sp. (algumas espécies desse gênero são utilizadas no telhado de construções rurais rústicas como madeira roliça) e Richardella macrophylla (usada em serrarias e com potencial para produção de carvão). Juntas, essas espécies representam 22,7\% do VIA.

$\mathrm{Na} \mathrm{FNM}_{1}$, as espécies com VIA $\geq 2,0 \%$ foram: Rinorea guianensis, Eschweilera coriacea, Protium cf. heptaphyllum, Sclerolobium sp. e Manilkara huberi - espécies de maior interesse econômico atualmente, pois são inúmeros os usos de sua madeira, sendo o principal em construção civil (PINHEIRO et al., 2007); Pouteria guianensis - além de seus frutos serem consumidos pelos animais, sua madeira pode ser utilizada em construção civil e para produção de carvão; Franchetella anibaefolia - frutos 
consumidos pela fauna; e Inga alba - frutos utilizados na alimentação humana e consumidos pela fauna, folhas e galhos usados para proteção e adubação do solo e madeira utilizada para produção de carvão.

A espécie Rinorea guianensis merece destaque, visto que apresentou o maior VIA na $\mathrm{FM}_{1} \mathrm{e}$ $\mathrm{FNM}_{1}$, porém sem valor de mercado, atualmente. Normalmente, espécies sem valor de mercado são suprimidas, no entanto, a eliminação dessas espécies deve ser de forma criteriosa. Além do mais, podem não ter uso madeireiro no momento, porém têm contribuição ecológica (ALMEIDA et al., 2012). Do ponto de vista da conservação, espécies sem valor atual para serraria e algumas dos gêneros Eschweilera, Protium, Guatteria e Inga exercem funções-chave no ecossistema que ocupam (PINHEIRO et al., 2007). Souza e Souza (2004) e Almeida et al. (2012) verificaram que Rinorea guianensis figura entre as espécies com maiores VIA em seus estudos.

A densidade de indivíduos estimados na $\mathrm{FM}_{2}$ foi de 472,5 ind.ha ${ }^{-1}$, com uma área basal de $26,2 \mathrm{~m}^{2} \cdot \mathrm{ha}^{-1}$ e volume de fuste de $324,1 \mathrm{~m}^{3} \cdot \mathrm{ha}^{-1}$ (Tabela 1). Na $\mathrm{FNM}_{2}$, encontraram-se 508 ind.ha 1 , com área basal de $28,9 \mathrm{~m}^{2} \cdot \mathrm{ha}^{-1} \mathrm{e}$ volume de fuste de $389,7 \mathrm{~m}^{3}$.ha ${ }^{-1}$ (Tabela 1). A altura dominante das árvores amostradas foi de 29,3 m e 24,9 m, respectivamente, para $\mathrm{FM}_{2}$ e $\mathrm{FNM}_{2}$.

Ao se avaliar o estoque de densidade, área basal e volume das duas condições $\left(\mathrm{FM}_{2}\right.$ e $\left.\mathrm{FNM}_{2}\right)$, observou-se que, após decorridos quatro anos do manejo florestal, houve uma redução de 2,7, 20,3 e 7,0\%, respectivamente, para área basal, volume e densidade, devido à extração de árvores de grande porte no tratamento $\mathrm{FM}_{2}$. A diferença entre tratamentos mostrou que $\mathrm{o}$ manejo florestal reduziu significativamente $(\mathrm{p}<0,05)$ o estoque de volume e área basal, no entanto, espera-se que essa diferença seja inexistente após o ciclo de corte de 25 anos instituído na área (Tabela 2). Esses resultados estão em consonância com os encontrados por Pinto et al. (2002) e Ribeiro et al. (2013) em outras áreas de floresta ombrófila densa.

Entre as 134 espécies identificadas na $\mathrm{FM}_{2}, 74,6 \%$ apresentaram $\mathrm{DA} \geq 1$ ind.ha ${ }^{-1}$. As espécies mais abundantes, em ordem decrescente (DA $\geq 11$ ind.ha $^{-1}$ ), foram Licania kunthiana Hook.f., Pouteria cladantha, Eschweilera coriacea, Protium cf. heptaphyllum, Nectandra sp., Pouteria guianensis, Bixa arborea Huber, Tetragastris altissima (Aubl.) Swart, Chamaecristas cleroxylon e Richardella macrophylla, que, juntas, representam 41,6\% da densidade absoluta total (Tabela 5). Essas espécies se distribuem de forma agrupada ou tendendo à agregação, exceto Tetragastris altissima, distribuída aleatoriamente.

Entre as 146 espécies inventariadas na $\mathrm{FNM}_{2}, 62,3 \%$ apresentaram DA $\geq 1$ ind.ha ${ }^{-1}$. As espécies de maior densidade absoluta (DA $\geq 13$ ind.ha $^{-1}$ ) foram Eschweilera coriacea, Nectandra sp., Bixa arborea, Protium cf. heptaphyllum, Licania kunthiana, Pouteria guianensis, Tetragastris altissima, Pouteria cladantha, Duguetia sp. e Richardella macrophylla, que, juntas, contribuíram com $37,1 \%$ da densidade total.

$\mathrm{Na} \mathrm{FM}_{2}$, de todos os indivíduos amostrados, $27,4 \%$ ocorreram no estrato inferior, $70,1 \%$ no estrato médio e 2,5\% no estrato superior. Esse fato foi observado por Souza e Souza (2004), em estudos sobre a estratificação vertical multivariada de uma floresta não explorada em Paragominas (PA), demonstrando que o estrato médio possuía o maior número de indivíduos.

$\mathrm{Na} \mathrm{FM}_{2}$, as espécies que apresentaram maiores valores de PSA foram Licania kunthiana, Eschweilera coriacea, Nectandra sp., Pouteria cladantha, Protium cf. heptaphyllum, Tetragastris altissima e Pouteria guianensis, que, juntas, representam 34,1\% da PSA total. $\mathrm{Na} \mathrm{FNM}_{2}$, os maiores valores de PSA foram de Bixa arborea, Manilkara bidentata (A.DC.) A.Chev., Myrciaria floribunda (H.West ex Willd.) O.Berg, Zygia racemosa (Ducke) Barneby \& J.W.Grimes, Sclerolobium paniculatum, Eschweilera grandiflora e Mezilaurus itauba Taubert ex Mez, totalizando 15,2\% da PSA total.

Myrciaria floribunda, Zygia racemosa, Sclerolobium paniculatum e Eschweilera grandiflora, apesar de estarem entre as maiores espécies com PSA na $\mathrm{FNM}_{2}$, não apresentam altos valores de VIA. Isso se deve ao fato de que essas espécies tiveram valores baixos nos outros parâmetros avaliados, como número total de indivíduos, dominância e frequência.

Entre as 134 espécies amostradas na $\mathrm{FM}_{2}$, apenas 9,7\% estão distribuídas em todos os estratos, entre elas destacaram-se Licania kunthiana, Pouteria cladantha, Pouteria guianensis, Manilkara huberi e Chamaecristas cleroxylone que estão entre as 15 espécies de maior VIA, 30,6\% distribuídas em dois estratos e $59,7 \%$ com indivíduos em apenas um estrato.

Das 146 espécies inventariadas na $\mathrm{FNM}_{2}$, apenas $9(6,2 \%)$ se encontram presentes nos três estratos, tais como Licania kunthiana, Pouteria guianensis e Manilkara huberi. Observou-se ainda que $38,9 \%$ das espécies apresentaram indivíduos em dois estratos e 56,3\% com árvores em apenas um estrato.

FLORESTA, Curitiba, PR, v. 45, n. 3, p. 465 - 476, jul. / set. 2015.

Vieira, D. dos S. et al.

ISSN eletrônico 1982-4688 / ISSN impresso 0015-3826 
Tabela 5. Estimativa dos parâmetros fitossociológicos das espécies com valor de importância ampliado (VIA $\geq 1,5$ ) para os tratamentos $\mathrm{FM}_{2}$ e $\mathrm{FNM}_{2}$, município de Placas, Pará.

Table 5. Estimation of phytosociological parameters of species with value larger importance (VIA $\geq$ 1.5) for $\mathrm{FM}_{2}$ and $\mathrm{FNM}_{2}$ treatments, Municipality of Placas, Pará.

\begin{tabular}{|c|c|c|c|c|c|c|c|c|}
\hline \multirow{2}{*}{ Espécies } & \multicolumn{2}{|c|}{$\mathbf{P}$} & \multicolumn{2}{|c|}{ FA (\%) } & \multicolumn{2}{|c|}{ DA (n.ha $\left.{ }^{-1}\right)$} & \multicolumn{2}{|c|}{ VIA (\%) } \\
\hline & $\mathbf{F M}_{2}$ & $\mathrm{FNM}_{2}$ & $\mathbf{F M}_{2}$ & $\mathbf{F N M}_{2}$ & $\mathbf{F M}_{2}$ & $\mathrm{FNM}_{2}$ & $\mathbf{F M}_{2}$ & $\mathbf{F N M}_{2}$ \\
\hline Licania kunthiana Hook.f. & $\mathrm{AG}$ & $\mathrm{AG}$ & 100 & 63,6 & 49,2 & 18 & 7,84 & 2,03 \\
\hline Pouteria cladantha Sandwith & AG & AG & 88,9 & 72,7 & 28,2 & 16,2 & 4,41 & 3,43 \\
\hline Eschweilera coriacea (DC.) S.A.Mori & TAG & AG & 88,9 & 72,7 & 20,8 & 30,2 & 3,81 & 3,62 \\
\hline Mezilaurus itauba Taubert ex Mez. & AG & - & 77,8 & - & 9,3 & - & 3,58 & - \\
\hline Nectandra sp. & AG & TAG & 77,8 & 90,9 & 18,3 & 23,0 & 3,09 & 2,59 \\
\hline Pouteria guianensis Aubl. & AG & AG & 66,7 & 90,9 & 14,4 & 17,4 & 2,76 & 3,23 \\
\hline Protium cf. heptaphyllum (Aubl.) Marchand & AG & AG & 77,8 & 72,7 & 18,9 & 18,6 & 2,71 & 2,47 \\
\hline Manilkara bidentata (A.DC.) A.Chev. & AG & - & 88,9 & - & 9,0 & - & 2,63 & - \\
\hline Manilkara huberi (Ducke) Chev. & AG & - & 88,9 & - & 9,2 & - & 2,40 & - \\
\hline Tetragastris altissima (Aubl.) Swart & $\mathrm{AL}$ & TAG & 77,8 & 100 & 12,2 & 17,3 & 2,27 & 2,55 \\
\hline Cassia scleroxylon Ducke & AG & - & 44,4 & - & 11,6 & - & 2,22 & - \\
\hline Sclerolobium $\mathrm{sp} 2$ & $\mathrm{AL}$ & - & 77,8 & - & 7,0 & - & 1,86 & - \\
\hline Bixa arborea Huber & AG & - & 44,4 & - & 13,3 & - & 1,81 & - \\
\hline Richardella macrophylla (Lam.) Aubrév. & TAG & AG & 66,7 & 45,5 & 9,4 & 12,8 & 1,80 & 4,10 \\
\hline Minquartia guianensis Aubl. & AG & - & 55,6 & - & 7,7 & - & 1,78 & - \\
\hline Glicoxylon pedicellatum Ducke & - & TAG & - & 9,1 & - & 0,9 & - & 2,80 \\
\hline Prieurella prieurii (A. DC.) Aubr. & - & TAG & - & 27,3 & - & 4,5 & - & 2,56 \\
\hline Pouteria caimito (Ruiz \& Pav.) Radlk. & - & AL & - & 18,2 & - & 0,5 & - & 2,02 \\
\hline Franchetella gougrijpii (Eyma) Aubrév. & - & AG & - & 27,3 & - & 4,6 & - & 2,03 \\
\hline Swartzia ingifolia Ducke & - & $\mathrm{AL}$ & - & 18,2 & - & 1,4 & - & 2,04 \\
\hline Chrysophyllum anomalum Pires & - & AG & - & 9,1 & - & 1,8 & - & 1,95 \\
\hline Rinorea guianensis Aubl. & - & TAG & - & 27,3 & - & 2,0 & - & 1,89 \\
\hline Subtotal & - & - & 1122 & 609 & 238,7 & 153,5 & 45 & 24 \\
\hline Total & - & - & 3689 & 4036 & 472,6 & 508,0 & 100 & 100 \\
\hline
\end{tabular}

P: índice de Payandeh; DA: densidade absoluta; FA: frequência absoluta; VIA: valor de importância ampliado; AG: agregado; TAG: tendência à agregação; $\mathrm{AL}=$ aleatório; $\mathrm{FM}_{2:}$ floresta manejada; $\mathrm{FNM}_{2}$ : floresta não manejada na comunidade São Mateus.

As sete espécies com maiores valores de VIA na $\mathrm{FM}_{2}$ (VIA > 2,7\%) foram Licania kunthiana (utilizada como alimento para caça e uso medicinal), Pouteria cladantha (espécie destinada à produção de energia e com frutos utilizados na alimentação de animais), Eschweilera coriacea, Nectandra sp., Protium cf. heptaphyllum e Mezilaurus itauba (possuem potencial madeireiro) e Pouteria guianensis (possui múltiplos usos, tais como madeireiro, alimento para caça e geração de energia).

$\mathrm{Na} \mathrm{FNM}_{2}$, as sete espécies com maiores VIA (VIA $\geq 2,56 \%$ ) foram, em ordem decrescente: Richardella macrophylla (uso madeireiro, frutífero e alimento para fauna local), Eschweilera coriacea e Nectandra sp. (fins madeireiros), Pouteria cladanta e Pouteria guianensis (usos já mencionados), Glicoxylon pedicellatum (destinada para fins medicinais e de energia) e Prieurella prieurii (frutos utilizados para alimento da fauna).

Entre as espécies analisadas com maiores VIA, verificou-se que Licania kunthiana apresentou o maior valor na $\mathrm{FM}_{2}(7,84 \%)$ e foi presente em todos os estratos. Segundo Souza e Souza (2004), espécies ocorrentes no estrato ou dossel superior e que possuem indivíduos, simultaneamente, nos estratos médio e inferior têm sustentabilidade ambiental. $\mathrm{Na} \mathrm{FNM}_{2}$, destacou-se Richardella macrophylla, por apresentar valor de $4,10 \%$, sendo ausente nos estratos inferior e superior. Entre as 15 espécies com maior VIA, apenas Protium cf. heptaphyllum e Eschweilera coriacea apresentaram indivíduos em todas as áreas estudadas. Gama et al. (2005) afirmam que Eschweilera coriacea está amplamente distribuída nas florestas de terra firme do estado do Pará. Entre as espécies mais exploradas no PA Moju I e II e que apresentam os maiores VIA, nas áreas manejadas $\left(\mathrm{FM}_{1}\right.$ e $\left.\mathrm{FM}_{2}\right)$, encontram-se Manilkara huberi, Mezilaurus itauba e Lecythis jarana, o que aponta para a importância ecológica dessas espécies e a sustentabilidade do manejo florestal executado. 


\section{CONCLUSÕES}

- A extração de madeira provocou alterações na abertura do dossel, mas não foram constatadas diferenças significativas no número de espécies e famílias nos tratamentos avaliados, sendo que diferenças florísticas quanto à diversidade entre sítios foram verificadas na mesma comunidade e entre comunidades. As espécies arbóreas inventariadas fazem parte de uma mesma tipologia florestal, e o manejo, mesmo de baixo impacto, afetou o padrão de distribuição espacial de algumas espécies arbóreas.

- As áreas apresentam elevado potencial para a exploração madeireira, desde que haja manutenção da diversidade de espécies e um estoque volumétrico apropriado às funções ecológicas da floresta.

- A colheita realizada na CSA não alterou os estoques totais de área basal, densidade e volume da área manejada, o que não foi observado na CSM, comprovando que o manejo florestal aplicado na CSA foi de baixo impacto, o que minimiza os danos às arvores remanescentes e garante a sustentabilidade da floresta. Na CSM, o manejo florestal não foi ambientalmente sustentável, entretanto, espera-se que ao fim do ciclo de corte o estoque de área basal e volume sejam repostos, o que irá garantir a sustentabilidade da floresta.

\section{REFERÊNCIAS}

ALMEIDA, L. S.; GAMA, J. R. V.; OLIVEIRA, A. S.; CARVALHO, J. O. P.; GONÇALVES, D. C. M.; ARAÚJO, G. C. Fitossociologia e uso múltiplo de espécies arbóreas em floresta manejada, Comunidade Santo Antônio, município de Santarém, Estado do Pará. Acta Amazonica, Manaus, v. 42, n. 2, p. 185 194, 2012.

ALVES, J. C. Z. O.; MIRANDA, I. S. Análise da estrutura de comunidades arbóreas de uma floresta Amazônica de terra firme aplicada ao manejo florestal. Acta Amazonica, Manaus, v. 38, n. 4, p. 657 666, 2008.

ANGIOSPERM PHYLOGENY GROUP. Na update of the Angiosperm Phylogeny Group classification for the orders and families of flowering plants: APG III. Botanical Journal of the Linnean Society, London, v. 16, p. 105 - 121, 2009.

BROWER, J. E.; ZAR, J. H. Field and laboratory methods for general ecology. W. M. C. Brow: Dubuque, 2. ed. 1984, $226 \mathrm{p}$.

ESPÍRITO-SANTO, F. D. B.; SHIMABUKURO, Y. E.; ARAGÃO, L. E. O. C.; MACHADO, E. L. M. Análise da composição florística e fitossociológica da Floresta Nacional do Tapajós com o apoio geográfico de imagens de satélites. Acta Amazonica, Manaus, v. 35, n. 2, p. 155 - 173, 2005.

FINOL, U. H. Nuevos parametros a considerar-se en el análises estructural de las selvas virgenes tropicales. Revista Forestal Venezoelana, Mérida, v. 14, p. 24 - 42, 1971.

FUNDO DE DESENVOLVIMENTO E AÇÃO COMUNITÁRIA (FUNDAC). Plano de Desenvolvimento Sustentável Moju I e II. Relatório Técnico. 153 p. 2005.

FRANCEZ, L. M. B.; CARVALHO, J. O. P.; JARDIM, F. C. S. Mudanças ocorridas na composição florística em decorrência da exploração florestal em uma área de floresta de terra firme na região de Paragominas, PA. Acta Amazonica, Manaus, v. 37, n. 2, p. 211 - 228, 2007.

GAMA, J. R. V.; RIBEIRO, A. S. S.; MORAES, A.; SANTOS, C. A. A.; MONTEIRO, R.; GOCH, Y. G. F. Estudo de impacto ambiental e proposta de desenvolvimento sustentável para o Assentamento Moju I e II. Relatório Técnico. Universidade Federal do Oeste do Pará (UFOPA), Santarém, 250 p. 2011.

GAMA, J. R. V.; SOUZA, A. L.; MARTINS, S. V.; SOUZA, D. R. Comparação entre florestas de várzea e terra firme do estado do Pará. Revista Árvore, Viçosa, v. 29, n. 4, p. 607 - 616, 2005.

GONÇALVEZ, F. G.; SANTOS, J. R. Composição florística e estrutura de uma unidade de manejo florestal sustentável na Floresta Nacional do Tapajós, Pará. Acta Amazonica, Manaus, v. 38, n. 2, p. 229 $-244,2008$.

FLORESTA, Curitiba, PR, v. 45, n. 3, p. 465 - 476, jul. / set. 2015.

Vieira, D. dos S. et al.

ISSN eletrônico 1982-4688 / ISSN impresso 0015-3826

DOI: $10.5380 /$ rf.v45i3.35584 
INSTITUTO BRASILEIRO DO MEIO AMBIENTE E DOS RECURSOS NATURAIS RENOVÁVEIS (IBAMA). Norma de Execução no 01 de 24 de abril de 2007. Brasília, 26 p. 2007.

KENT, M.; COKER, P. Vegetation description analyses. Behaven: London, 2 ed. 1992, 363 p.

MACHADO, F. S. Manejo de produtos florestais não madeireiros: um manual com sugestões para o manejo participativo em comunidades da Amazônia. Rio Branco: PESACRE e CIFOR, 1. ed. 2008. $105 \mathrm{p}$.

MARTINS, S. S.; COUTO, L.; MACHADO, C. C.; SOUZA, A. L. Efeito da exploração florestal seletiva em uma Floresta Estacional Semidecidual. Revista Árvore, Viçosa, v. 27, n. 1, p. 65 - 70, 2003.

MATTEUCCI, S. D.; COLMA, A. Metodologia para el estúdio de la vegetación. OEA: Washington, 1. ed. 1982, 167 p.

MISSOURI BOTANICAL GARDEN (MOBOT). Nomenclatural Data Base. Disponível em: <http://mobot.mobot.org/cgi-bin/search_vast>. Acesso em 11/06/2014. 2014.

PINHEIRO, K. A. O.; CARVALHO, J. O. P.; QUANZ, B.; FRANCEZ, L. M. B.; SCHWARTZ, G. Fitossociologia de uma área de preservação permanente no leste na Amazônia: indicação de espécies para recuperação de áreas alteradas. Revista Floresta, Curitiba, v. 37, n. 2, p. 175 - 187, 2007.

PINTO, A. C. M.; SOUZA, A. L.; SOUZA, A. P.; MACHADO, C. C.; MINETTE, L. J.; VALE, A. B. Análise de danos de colheita de madeira em floresta tropical úmida sob regime de manejo florestal sustentado na Amazônia ocidental. Revista Árvore, Viçosa, v. 26, n. 4, p. 459 - 466, 2002.

RIBEIRO, R. B. S.; GAMA, J. R. V.; MARTINS, S. V.; MORAES, A.; SANTOS, C. A. A.; CARVALHO, A. D. Estrutura florestal em projeto de assentamento, comunidade São Mateus, município de Placas, Pará, Brasil. Revista Ceres, Viçosa, v. 60, n. 5, p. 610 - 620, 2013.

SOUZA, A. L.; SOARES, C. P. B. Florestas nativas: estrutura, dinâmica e manejo. Viçosa, MG: Editora UFV, 1. ed. 2013, 322 p.

SOUZA, D. R.; SOUZA, A. L. Estratificação vertical em floresta ombrófila densa de terra firme não explorada, Amazônia Oriental. Revista Árvore, Viçosa, v. 28, n. 5, p. 691 - 698, 2004.

VIEIRA, D. S.; GAMA, J. R. V.; RIBEIRO, R. B. S.; XIMENES, L. C.; CORRÊA, V. V.; ALVES, A. F. Comparação estrutural entre floresta manejada e não manejada na comunidade Santo Antônio, estado do Pará. Revista Ciência Florestal, v. 24, n. 4, p. 1067 - 1074, 2014.

XIMENES, L. C.; VIEIRA, D. S.; GAMA, J. R. V.; SILVA-RIBEIRO, R. B.; CORREAA, V. V.; ALVES, A. F. Estrutura de floresta manejada por comunitários na FLONA Tapajós. In: $5^{\circ}$ Simpósio Latinoamericano sobre manejo florestal (Sustentabilidade florestal), 2., 2011, Santa Maria. Anais... Santa Maria, 2011. p. $686-693$. 